\title{
Exponentially enhanced quantum metrology
}

\author{
S. M. Roy ${ }^{1,2}$ and Samuel L. Braunstein ${ }^{1}$ \\ ${ }^{1}$ Computer Science, University of York, York YO 10 5DD, United Kingdom \\ ${ }^{2}$ Department of Theoretical Physics, Tata Institute of Fundamental Research, \\ Homi Bhabha Road, Mumbai 400005, India
}

(Dated: October 16, 2018)

\begin{abstract}
We show that when a suitable entanglement generating unitary operator depending on a parameter is applied on $N$ qubits in parallel, a precision of order $2^{-N}$ in estimating the parameter may be achieved. This exponentially improves the precision achievable in classical and in quantum nonentangling strategies.
\end{abstract}

PACS numbers: 03.65.Ta, 03.67.Lx,06.20.Dk,42.50.St

Keywords: quantum metrology, quantum control, quantum computation

The Mandelstam-Tamm version of the time energy uncertainty relation [1] and its rigorous developments [2] form the basis of quantum enhanced methods for parameter estimation such as those used in evolving frequency standards [3]. Giovannetti, Lloyd and Maccone 4] have recently done beautiful work to introduce a general framework to classify methods of such quantum enhancement. A unitary transformation depending on the parameter to be estimated is applied to a suitably prepared system of $N$ probes and then an appropriate observable is measured. A separable initial state is labelled $\mathrm{C}$ and an entangled state Q. Similarly, measured observables which are direct products of individual qubit observables are designated $\mathrm{C}$ and those which introduce entanglement are designated Q. Thus the strategies of "parallel" measurement on $N$ qubits are classified as CC, CQ, QC, QQ. Their result is that quantum metrology in the strategies $\mathrm{CC}$ and $\mathrm{CQ}$ only achieve the classicalprecision limit of order $\epsilon / \sqrt{N}$, where $\epsilon$ is the dispersion of measurement results for each probe; but the quantum metrology strategies QC and QQ can achieve a precision of order $\epsilon / N$ if the probes are prepared in a suitable entangled state. In contrast, for $N$ sequential measurements on a single probe to achieve the same precision of order $1 / N$ requires a running time and hence duration of quantum coherence to be $N$ times longer.

We propose here a parallel strategy which exploits the Hilbert space of $N$ probes more fully than in previous work [4] and thereby attains an exponentially enhanced precision. In discussing parallel strategies previous work have only considered applying on the prepared probes a unitary operator which is a direct product of $N$ unitary operators each acting on a single probe. We will show here that if instead we consider applying on the probes, an entanglement generating unitary operator $U=e^{-i \theta H}$ which cannot be written as a direct product of one-probe operators, then we can obtain an exponentially enhanced precision in estimating the parameter $\theta$. The fundamental reason for this improvement is that there are an exponentially large number of mutually commuting observables for the $\mathrm{N}$-probe system whereas the number of mutually commuting single-probe operators is only of order $N$. For instance, for the $N$-qubit system each qubit can- not have more than one commuting observable and hence there are exactly $N$ commuting observables of the form $\mathbb{1} \otimes \cdots \otimes \mathbb{1} \otimes A^{j} \otimes \mathbb{1} \otimes \ldots \mathbb{1}$ each of which acts nontrivially on only the $j$ th qubit, with $j=1,2, \ldots, N$. We construct sets of $2^{N-1}$ mutually commuting Hermitian operators each of the form $A^{1} \otimes A^{2} \otimes \cdots \otimes A^{N}$ which acts non-trivially on all the $N$ qubits. The unitary entanglement generating operator is chosen to be a product (but not a direct product) of the exponentials of these Hermitian operators times $-i \theta$. In this way, we fully exploit the quantum parallelism which is at the heart of exponential violations of local realism [ $[\underline{5}$ and of the well-known exponential speed-up achieved in certain quantum computation tasks [6]. For the probe system of $N$ qubits we obtain the best possible precision of order $\epsilon 2^{-N}$ dictated by the uncertainty principle for such entanglement-generating unitary operators. The previous precision limit of order $\epsilon / N$ is the best possible for unitary operators which are direct products. We thus obtain a new characterization of eight parallel quantum metrology strategies as $X Y Z$ where each of $X, Y$ and $Z$ can be Q or C. See Fig. 1. Here $X$ and $Z$ specify presence or absence of entanglement in the probes and observables respectively as in [4], and the new label $Y$ specifies whether the unitary operator $U$ applied on the probes is an entanglement-generating operator or a direct product.

In our parallel strategy, a precision of order $\epsilon 2^{-N}$ is obtained using an entanglement generating operator that is a product of $2^{N-1}$ mutually commuting unitary operators, but entanglement at the probe preparation stage and measurement stage are inessential. To obtain the same precision in the sequential strategy of Ref. 4 one needs an exponentially large number of applications of a unitary operator on a single qubit; hence the time for which quantum coherence has to be maintained will be longer by a factor of order $2^{N}$. The increase in precision we achieve with respect to the parallel strategy of Ref. 4 is however at the cost of a "Hamiltonian" or "generator" of $U$ which might be more difficult to implement experimentally. Here again, quantum mechanics just might come to the rescue. We propose a quantum-optics model of laser light interacting with an $N$-qubit system, say a polyatomic molecule, via a generalized Jaynes-Cummings in- 


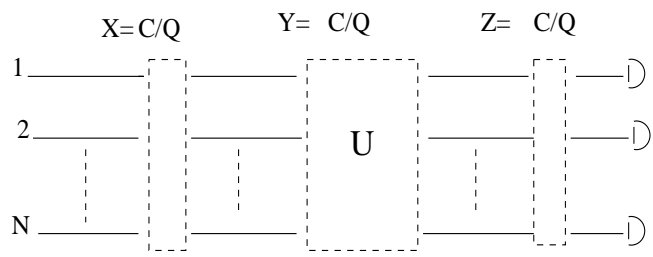

FIG. 1: The eight parallel strategies $X Y Z$ for estimation of a parameter occurring in an unitary operator $U$ applied to $N$ probes classified according to types of state preparation,unitary operation, and measurement. Each of $X, Y, Z$ takes values $C$ or $Q . \quad Y=Q$ if $U$ is an entanglement generating operator (as considered in this paper) and $Y=C$ otherwise. $X, Z$ specify presence of entanglement $(Q)$ or absence of entanglement $(C)$ in the prepared probe state and in the measured operator respectively.

teraction which, in principle, could achieve the exponentially enhanced precision. The practical implementability of this specific model is at the moment unclear, but would be an interesting subject for further theoretical and experimental investigation.

Quantum limits on precision: We recall first the Mandelstam-Tamm uncertainty relations. Suppose we apply an unitary operator $U=e^{-i \theta H}$ to a probe state $|\phi\rangle$ where $H$ is a self-adjoint operator and $\theta$ a parameter to be estimated. We obtain the state

$$
|\psi(\theta)\rangle=U|\phi\rangle
$$

and then measure the observable $X$ on this state with a view to estimating the parameter $\theta$. Schwarz inequality yields

$$
\begin{aligned}
\Delta X \Delta H & \geq|\langle\psi(\theta)|[H, X] /(2 i)| \psi(\theta)\rangle| \\
& =\frac{1}{2}\left|\frac{\partial\langle\psi(\theta)|X| \psi(\theta)\rangle}{\partial \theta}\right|,
\end{aligned}
$$

where $\Delta X, \Delta H$ denote the dispersions in $X, H$ respectively. The resulting precision in estimating $\theta$ is thus given by the uncertainty relation

$$
\delta \theta \equiv \Delta X /\left|\frac{\partial\langle\psi(\theta)|X| \psi(\theta)\rangle}{\partial \theta}\right| \geq \frac{1}{2 \Delta H} .
$$

In the case when the parameter $\theta$ is estimated using an estimator $\theta_{\text {est }}$ and the estimation is repeated $\nu$ times, the Cramer-Rao bound [7] was used in Ref. 2 to prove the generalized uncertainty relation for the error estimate

$$
\delta_{\nu} \theta \equiv\left\langle\left(\theta_{\mathrm{est}} /\left|\frac{\partial\left\langle\theta_{\mathrm{est}}\right\rangle_{\mathrm{av}}}{\partial \theta}\right|-\theta\right)^{2}\right\rangle_{\mathrm{av}}^{1 / 2} \geq \frac{1}{2 \Delta H \sqrt{\nu}},
$$

where $\langle\cdots\rangle_{\text {av }}$ denotes statistical average. The quantum limits on precision are obtained by noting that

$$
\Delta H \leq \frac{1}{2}\left(\lambda_{\max }-\lambda_{\min }\right)
$$

where $\lambda_{\max }, \lambda_{\min }$ denote respectively the maximum and minimum eigenvalues of $H$ which we assume to be finite. Hence, the Mandelstam-Tamm [1] and Braunstein-Caves 2] quantum limits on precision are given respectively by

$$
\delta \theta \geq \frac{1}{\left(\lambda_{\max }-\lambda_{\min }\right)},
$$

and

$$
\delta_{\nu} \theta \geq \frac{1}{\left(\lambda_{\max }-\lambda_{\min }\right) \sqrt{\nu}} .
$$

It is clear that quantum parallel strategies to improve precision should aim to maximize $\Delta H$ on the $N$-probe quantum state.

Parallel strategies for $N$ qubits: Consider first, as in [4] the operator $H$ to be a direct sum of the operators $H^{j}$ acting on the $j$-th probe, each $H^{j}$ having the same maximum dispersion $\Delta H^{j} \leq d$. Then

$$
\Delta \bigoplus_{j=1}^{N} H^{j} \leq N d
$$

As noted in Ref. 4 this maximum dispersion is in fact reached when we choose the $N$-qubit state to be an equally weighted superposition of the eigenvectors of $H$ with maximum and minimum eigenvalues. With $\lambda_{\max }=$ $N \lambda_{M}$ and $\lambda_{\min }=N \lambda_{m}$ where $\lambda_{M}$ and $\lambda_{m}$ are respectively the maximum and minimum eigenvalues of each $H^{j}$ we get the above equation with $d=\left(\lambda_{M}-\lambda_{m}\right) / 2$. Note for purposes of comparison, that the dispersion of a sum of $K$ classical variables $H_{\mathrm{cl}}^{j}$ with a factorized joint probability distribution is given by

$$
\Delta \sum_{j=1}^{K} H_{\mathrm{cl}}^{j}=\sqrt{\sum_{j=1}^{K}\left(\Delta H_{\mathrm{cl}}^{j}\right)^{2}}
$$

The maximum quantum dispersion is thus $\sqrt{N}$ times the classical value when $K=N$ and $\Delta H_{\mathrm{cl}}^{j}=d$. This has been exploited in Ref. 4. We now show that the dispersion of $H$ for $N$ qubits can be made exponentially larger by appropriate choice of $H$. Consider, the operator identity

$$
\bigotimes_{j=1}^{N}\left(\sigma_{x}+i \sigma_{y}\right)^{j}=H+i A
$$

where $\sigma_{x}^{j}$ and $\sigma_{y}^{j}$ are Pauli matrices for the $j$-th qubit, and $H$ and $i A$ denote respectively the Hermitian and antiHermitian parts of the operator on the left-hand side of the equation. Explicit expressions for $H$ and $A$ are conveniently stated in terms of the matrices $\sigma^{j}( \pm 1)$ defined by

$$
\sigma^{j}(+1) \equiv \sigma_{x}^{j}, \quad \sigma^{j}(-1) \equiv \sigma_{y}^{j}
$$


We obtain

$$
H=\sum_{\substack{r_{1}, r_{2}, \ldots, r_{N}= \pm 1 \\ N_{-}=\text {even }}} H\left(r_{1}, r_{2}, \ldots, r_{N}\right)
$$

where

$$
H\left(r_{1}, r_{2}, \ldots, r_{N}\right) \equiv(-1)^{N_{-} / 2} \bigotimes_{j=1}^{N} \sigma^{j}\left(r_{j}\right)
$$

and

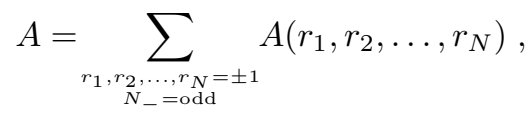

where

$$
A\left(r_{1}, r_{2}, \ldots, r_{N}\right) \equiv(-1)^{\left(N_{-}-1\right) / 2} \bigotimes_{j=1}^{N} \sigma^{j}\left(r_{j}\right)
$$

and finally

$$
N_{-}=\sum_{j=1}^{N} \frac{1}{2}\left(1-r_{j}\right)
$$

is just the number of $j$ 's with $r_{j}=-1$ or the number of $\sigma_{y}^{j}$ 's in the $N$-fold product of Pauli matrices in $H$ and $A$. Both $H$ and $A$ are sums of $2^{N-1}$ products of Pauli matrices, each product having eigenvalues \pm 1 and hence maximum dispersion

$$
\Delta \bigotimes_{j=1}^{N} \sigma^{j}\left(r_{j}\right) \leq 1
$$

The standard anti-commutation rules between Pauli matrices leads to

$$
\sigma^{j}\left(r_{j}\right) \sigma^{j}\left(r_{j}^{\prime}\right)=\sigma^{j}\left(r_{j}^{\prime}\right) \sigma^{j}\left(r_{j}\right) r_{j} r_{j}^{\prime}
$$

for $r_{j}, r_{j}^{\prime}= \pm 1$. Note that $r_{1} r_{2} \ldots r_{N}=(-1)^{N_{-}}$. The anti-commutation rules then imply that the set of $2^{N-1}$ products of Pauli matrices occurring in $H$ (or $A$ ) constitutes a set of mutually commuting observables. Hence

$$
e^{-i \theta H}=\prod_{\substack{r_{1}, r_{2}, \ldots, r_{N}= \pm 1 \\ N_{-}=\text {even }}} e^{-i \theta H\left(r_{1}, r_{2}, \ldots, r_{N}\right)}
$$

and

$$
e^{-i \theta A}=\prod_{\substack{r_{1}, r_{2}, \ldots, r_{N}= \pm 1 \\ N_{-}=\text {odd }}} e^{-i \theta A\left(r_{1}, r_{2}, \ldots, r_{N}\right)}
$$

Further any of these $2^{N-1}$ observables in $H$ anticommutes with any of the $2^{N-1}$ observables occurring in $A$. In contrast with the parallel strategy in Ref. 4, we now have

$$
\Delta H \leq 2^{N-1}, \quad \Delta A \leq 2^{N-1} .
$$

Interestingly, and in contrast with Ref. 4 the maximum dispersions of $H$ and $A$ are now reached in the separable $N$-qubit states

$$
|\uparrow \uparrow \ldots \uparrow \uparrow\rangle, \quad|\downarrow \ldots \ldots \downarrow\rangle,
$$

each of which has

$$
\Delta H=2^{N-1}, \quad \Delta A=2^{N-1},
$$

where we have denoted the eigenstates of $\sigma_{z}$ with eigenvalues +1 and -1 by $|\uparrow\rangle$ and $|\downarrow\rangle$ respectively.

For comparison, the maximum classical dispersion for the sum of $2^{N-1}$ classical variables with factorized probability distribution, each variable having maximum dispersion 1 , would be $2^{(N-1) / 2}$.

Parallel strategy CQC with exponentially enhanced precision: We start from one of the factorized $N$-qubit states given above and apply one of the unitary operators $U=e^{-i \theta H}$ or $U=e^{-i \theta A}$ given above. In particular, we obtain

$$
\begin{aligned}
& \left|\psi_{H}(\theta)\right\rangle=e^{-i \theta H}|\uparrow \uparrow \ldots \uparrow \uparrow\rangle \\
= & \cos \left(2^{N-1} \theta\right)|\uparrow \ldots \uparrow\rangle-i \sin \left(2^{N-1} \theta\right)|\downarrow \ldots \downarrow\rangle ; \\
& \left|\psi_{A}(\theta)\right\rangle=e^{-i \theta A}|\uparrow \uparrow \ldots \uparrow \uparrow\rangle \\
= & \cos \left(2^{N-1} \theta\right)|\uparrow \ldots \uparrow\rangle+\sin \left(2^{N-1} \theta\right)|\downarrow \ldots \downarrow\rangle .
\end{aligned}
$$

We may now measure the probability that all qubits are in the up-state given by the expectation value of the direct product of projection operators

$$
X=\bigotimes_{j=1}^{N} \frac{1}{2}\left(\mathbf{1}+\sigma_{z}^{j}\right)
$$

We obtain, for example for the state $\left|\psi_{H}(\theta)\right\rangle$

$$
\langle X\rangle=\frac{1}{2}\left[1+\cos \left(\theta 2^{N}\right)\right], \quad \Delta X=\frac{1}{2}\left|\sin \left(\theta 2^{N}\right)\right| .
$$

Hence, the quantum precision of estimating $\theta$ is given by

$$
\delta \theta=2^{-N}
$$

which achieves the best allowed by the uncertainty relation since $\Delta H=2^{N-1}$. A similar precision is obtained by using the state $\left|\psi_{A}(\theta)\right\rangle$. It is clear that we only needed to use the parallel strategy $C Q C$ (no entanglement in the probe-preparation or measurement stage, but an entanglement-generating unitary operator applied on the probes) to obtain this exponential enhancement of precision. 
A generalized Jaynes-Cummings model: We propose a quantum optics model of interaction of laser light with $N$ qubits which could, in principle, be used to obtain exponentially enhanced precision. We apply a unitary operator to the infinite-dimensional vector space which implies an entanglement generating 'operation' on the reduced density operator for $N$ qubits.

Suppose we shine laser light of frequency $\Omega \approx N \omega$ on an $N$-atom molecule each atom of which can be approximated to be a two-level atom or qubit with level separation $\hbar \omega$. We assume that on irradiation, the molecule does not dissociate but gets excited to a higher level in which each atom is excited to the higher level. The second quantized interaction Hamiltonian is assumed to be

$$
\begin{gathered}
\hat{H}=\hat{H}_{0}+\hat{H}_{I}, \quad \hat{H}_{0}=\frac{\omega}{2} \sum_{j=1}^{N} \hat{\sigma}_{z}^{j}+\Omega \hat{a}^{\dagger} \hat{a}, \\
\hat{H}_{I}=\frac{g}{2}\left[\hat{a} \bigotimes_{j=1}^{N}\left(\hat{\sigma}_{x}+i \hat{\sigma}_{y}\right)^{j}+\text { h.c. }\right] .
\end{gathered}
$$

Here h.c. denotes Hermitian conjugate, $\hat{a}$ and $\hat{a}^{\dagger}$ denote annihilation and creation operators for photons of frequency $\Omega, \frac{1}{2}\left(\hat{\sigma}_{x}+i \hat{\sigma}_{y}\right)^{j}$ denotes the level raising operators for the $j$-th qubit and $g$ is a real coupling constant. It is an exactly solvable Hamiltonian with eigenvalues

$$
\lambda_{ \pm}=\Omega\left(n+\frac{1}{2}\right) \pm \frac{\Omega_{1}}{2},
$$

where $n=0,1,2, \ldots$ and

$$
\Omega_{1}=\sqrt{(\omega N-\Omega)^{2}+g^{2}(n+1) 2^{2 N}} .
$$

The corresponding eigenstates are,

$$
\begin{gathered}
\frac{1}{\sqrt{2}}\left(\alpha_{ \pm}|n\rangle\left|\phi_{0}\right\rangle+\beta_{ \pm}|n+1\rangle\left|\phi_{1}\right\rangle\right), \\
\left|\phi_{0}\right\rangle \equiv|\uparrow \ldots \uparrow\rangle, \quad\left|\phi_{1}\right\rangle \equiv|\downarrow \ldots \downarrow\rangle,
\end{gathered}
$$

$$
\begin{aligned}
\alpha_{ \pm} & \equiv \sqrt{1 \pm(\omega N-\Omega) / \Omega_{1}} \\
\beta_{ \pm} & \equiv \pm \sqrt{1 \mp(\omega N-\Omega) / \Omega_{1}}
\end{aligned}
$$

and $\hat{a}^{\dagger} \hat{a}|n\rangle=n|n\rangle$. Interesting physics about Rabi oscillations between up and down qubit states may be read off from these equations even off-resonance, i.e., $\Omega \neq N \omega$. For the present we specialize to $\Omega=N \omega$ which implies $\left[\hat{H}_{0}, \hat{H}_{I}\right]=0$. We deduce that for $r=0$ and $r=1$

$$
\begin{aligned}
& e^{-i t \hat{H}}|n+r\rangle\left|\phi_{r}\right\rangle=e^{-i t(n+1 / 2) N \omega} \\
& \times\left[\cos \left(2^{N-1} \theta\right)|n+r\rangle\left|\phi_{r}\right\rangle\right. \\
& \left.\quad-i \sin \left(2^{N-1} \theta\right)|n+1-r\rangle\left|\phi_{1-r}\right\rangle\right],
\end{aligned}
$$

where $\theta=t g \sqrt{n+1}$. Thus the reduced density operator for the $N$ qubits obtained by tracing over the photon states undergoes the positivity and trace preserving transformation

$$
\hat{\rho}(t)=\sum_{r=0,1} \hat{h}_{r} \hat{\rho}(0) \hat{h}_{r}^{\dagger}, \quad \hat{h}_{r}=e^{-i \theta H}\left|\phi_{r}\right\rangle\left\langle\phi_{r}\right|,
$$

where $H$ is the $N$-qubit spin operator given by Eq. (10). For measurement of the observable $X$ of Eq. (26) with $\langle X\rangle=\operatorname{tr} \hat{\rho}(t) X$ we obtain exactly the same results on precision as in Eqs. (27) and (28) of the previous section. Investigation of practical implementability of this model for achieving exponentially enhanced precision is an important remaining task.

In conclusion, we have shown that the parameter estimation associated with suitable entanglement-generating unitary operators may lead to an exponential enhancement of accuracy over both classical schemes and nonentangling quantum schemes. The exciting thing is that unlike quantum computation, quantum metrology might not face formidable problems of fighting decoherence if a suitable interaction Hamiltonian can be found. We have shown that the suggested strategy may be implemented in principle using a generalized Jaynes-Cummings interaction.

This work is funded in part by EPSRC grant EP/D500354/1. SLB currently holds a Wolfson - Royal Society Research Merit award.
[1] L. Mandelstam and I. G. Tamm, J. Phys. USSR 9, 249 (1945); A. Messiah, Quantum Mechanics, (North Holland, Amsterdam, 1961) Vol. I, pp. 319 and 320.

[2] S. L. Braunstein and C. M. Caves, Phys. Rev. Lett. 72, 3439 (1994); S. L. Braunstein, C. M. Caves and G. J. Milburn, Ann. Phys. (N.Y.) 247, 135 (1996).

[3] J. J. Bollinger, W. M. Itano, D. J. Wineland and D. J. Heinzen, Phys. Rev. A 54, R4649 (1996).

[4] V. Giovannetti, S. Lloyd and L. Maccone, Phys. Rev. Lett. 96, 010401 (2006); Science 306, 1330 (2004); Phys. Rev. A 65, 022309 (2002); Nature (London) 412, 417 (2001).
[5] N. D. Mermin, Phys. Rev. Lett. 65, 1838 (1990); S. M. Roy and V. Singh, Phys. Rev. Lett. 67, 2761 (1991).

[6] P. W. Shor, in Proceedings of the 35th Annual Symposium on Foundations of Computer Science, edited by S. Goldwasser (IEEE Computer Society, Los Alamos, CA, 1994), p. 124 ; D. S. Abrams and S. Lloyd, Phys. Rev. Lett. 83, 5162 (1999).

[7] H. Cramér, Mathematical Methods of Statistics, (Princeton University Press, 1946). 